\title{
La construcción social de la maternidad en México y las mujeres que deciden no procrear
}

\author{
The social construction of motherhood in Mexico \\ and women who decide not to procreate
}

JESSICA HERNÁNDEZ GONZÁLEZ*
Maestrante en psicoterapia sistémica
Instituto Bateson de Psicoterapia sistémica.

Recibido: $18 / 6 / 2019$

Aceptado: $14 / 10 / 2019$

doi: https://doi.org/10.20318/femeris.2020.5153

\begin{abstract}
Resumen. El presente artículo" hace referencia a las implicaciones socioculturales que tiene el ser mujer y no ser madre dentro del contexto mexicano, caracterizado por tener extremadamente arraigado el modelo de familia tradicional. En México, toda estructura familiar que se salga de la heteronormatividad, incluyendo a familias homoparentales, monoparentales, parejas LGBTTTIQ+ y parejas sin hijos, es cuestionada, juzgada y criticada.

El abordaje se realiza principalmente desde la epistemología feminista, ya que, a partir de la política y la crítica, visibiliza las relaciones desiguales de poder que existen entre hombres y mujeres, así como los roles de género que se han impuesto socio-históricamente a cada uno, las cuales son legitimadas por los discursos de la ciencia, la religión y el patriarcado, generando que los hombres continúen siendo los principales ocupantes de los espacios públicos y las mujeres de los espacios privados como lo es el hogar y lo que éste conlleva esencialmente: la maternidad. Como expone Saletti, un objetivo de la teoría feminista es "analizar la construcción de los discursos sociales sobre las mujeres" (2008, p. 169), siendo uno de ellos el de la maternidad. De igual manera, señala que "al revelar el carácter construido [de la práctica materna], demuestra que el imaginario social sobre la misma está configurado por diversas representaciones que identifican la maternidad con la feminidad proporcionando un ideal común para todas las mujeres" (2008, p.170). Es por esto que el artículo aborda temas como roles de género, maternidad, ciencia, patriarcado y la manera en que estos aspectos repercuten o influyen en las mujeres que han decidido no ser madres.
\end{abstract}

Palabras clave: maternidad, construcción social, género, poder, mujeres sin hijos. .

Abstract. This article*** refers to the sociocultural implications of being a woman and not being a mother in the Mexican context, characterized by having an extremely traditional family model. In Mexico, any family structure that gets out of the heteronormativity, including homoparental families, single parents, LGBTTTIQ+ couples and couples without children, is questioned, judged and criticized.

\footnotetext{
*jesshg27@gmail.com

${ }^{* *}$ Este artículo surge a partir de la tesis "Parejas que no quieren tener hijos: narrativas y discursos dominantes sobre la maternidad y la familia tradicional en México", realizado por la autora para obtener el grado de Maestra en Psicoterapia Sistémica.

${ }^{* * *}$ This article is based on the thesis "Couples who do not want children: narratives and dominant discourses about motherhood and traditional family in Mexico", made by the author to obtain the Master's Degree in Systemic Psychotherapy.
} 
The approach is made mainly from the feminist epistemology, since, from politics and criticism, it makes visible the unequal power relations that exist between men and women, as well as the gender roles that have been imposed socio-historically on each one, which are legitimized by the discourses of science, religion and patriarchy, generating that men continue to be the main occupants of the public spaces and women of the private spaces such as the home and what it essentially entails: motherhood. As Saletti explains, one goal of feminist theory is "to analyze the construction of social discourses about women" (2008, p. 169), one of them being motherhood. She also states that "by revealing the constructed character [of maternal practice], it demonstrates that the social imaginary about it, is shaped by diverse representations that identify motherhood with femininity, providing a common ideal for all women" $(2008$, p. 170). That is why the article addresses issues such as gender roles, motherhood, science, patriarchy and how these aspects impact or influence women who have decided not to be mothers.

Keywords: motherhood, social construction, gender, power, child-free women.

\section{Introducción}

Socio-históricamente se ha considerado que la mujer posee un carácter innato y biológico para ser madre, y más aún, ser una "buena" madre, lo que conlleva el cuidado, afecto, cariño, atención, preocupación, etcétera hacia los hijos. Bajo esa lógica, nacer mujer significa, implícitamente, ser madre en algún momento y, si se decide salir de esta regla social, se entra automáticamente al grupo de las mujeres "diferentes". Palomar (2005) menciona que a este grupo pertenecen las madres que tienen otras prioridades además de sus hijos, las madres que están institucionalizadas en hospitales psiquiátricos o en cárceles, o las mujeres que han decidido no ejercer el rol de madre.

En el estudio sociohistórico que realiza Lagarde (1990) sobre las mujeres, menciona que la filosofía feminista de Simone de Beauvoir (1949) sirvió como referente para los futuros movimientos feministas, en donde inclusive hoy en día, se busca reflexionar sobre la situación del sexo femenino, la equidad de género, así como derogar los estereotipos de género dañinos que se han impuesto sobre la mujer y generar nuevas identidades femeninas, más allá de la clásica identidad-maternidad.

\section{Metodología}

Para este artículo se utilizó la metodología de revisión bibliográfica, ya que a partir de distintas búsquedas ${ }^{1}$ se identificó que existe abundante teoría respecto a la maternidad y a las mujeres que no pueden ser madres, sin embargo, no hay suficiente investigación sobre las mujeres que no desean tener hijos. Desde este punto, se puede observar cómo la literatura científica también es parte en la reproducción de discursos los cuales imponen la maternidad. En este sentido, el presente trabajo contribuye a visibilizar básicamente dos puntos: 1) bibliografía que respalda la afirmación de que existen distintas instituciones y disciplinas que utilizan el poder para desarrollar e implantar en la sociedad verda-

\footnotetext{
${ }^{1}$ Se utilizaron bases de datos como Google Académico, REDALYC, SciELO, Dialnet, Academia.edu, Springer Link, entre otros.
} 
des dominantes respecto a la procreación y, 2) sustento teórico del por qué la maternidad, más que ser una cuestión biológica, es una construcción social.

\section{Estereotipos de género}

El género es un concepto clave dentro de la teoría feminista. Género es una construcción social sistemática de lo masculino y lo femenino que está poco (o nada) determinada por la biología, presente en todas las sociedades, y que permea todas las dimensiones de la vida social y privada (Harding, 1983). Con este concepto ha sido posible comenzar a estudiar cómo la sociedad determina de manera crucial las formas masculinas y femeninas en que se comportan las personas dependiendo del período histórico. Género es una definición cultural que se traduce, entre otras cosas, en una identidad que los individuos adquieren a través de la socialización, y que determina la forma en que se relacionan con la naturaleza y el mundo social que los rodea (Lamas, 1986; Lerner, 1986). Al analizar este término y su contenido, se puede observar que la sociedad en la que vivimos está organizada de una forma primordialmente masculina, donde los estereotipos de género privilegian al hombre.

Dentro de la construcción social de la maternidad, el género es una constante. Los roles de género, los estereotipos y la maternidad se relacionan unos con otros y se gestan desde la infancia, basta con prestar atención a los adjetivos con los que los niños son descritos: valientes, fuertes, aventureros, protectores, atrevidos, deportistas, inteligentes, etcétera, mientras que a las niñas se les adjudican las características "femeninas": cuidadoras, amorosas, sentimentales, delicadas. En pocas palabras, a los niños se les educa para ser proveedores y a las niñas para ser madres. Morell (1994) indica que las mujeres que deciden no ser madres, porque quieren ser autónomas y libres de responsabilidades maternales, son cuestionadas frecuentemente ya que estas actitudes son, dentro del imaginario colectivo, propias de los hombres. Una mujer que tiene esas actitudes puede ser descrita como masculina y de moral cuestionable.

Berger y Luckmann refieren que los fenómenos como los estereotipos de género se crean y se construyen dentro de la cotidianeidad, generando la "realidad de la vida cotidiana" (1968, p. 34). Ésta se da por establecida y es aceptada por los demás, normalizando la realidad. Esto es lo que sucede con los estereotipos de género relativos a la maternidad, que son decretados por el conocimiento experto-patriarcal, introduciéndolos en la realidad cotidiana, logrando a través de las relaciones de poder (subrepticias, en abundantes casos) que sea aceptada por la mayoría y cuestionada por muy pocos.

\section{Espacio privado: maternidad y crianza}

A lo largo de la historia, las mujeres han desempeñado roles relacionados con la crianza y el cuidado del espacio denominado "hogar", así como de las personas que lo habitan, especialmente hijos y pareja. Se sigue reproduciendo el mito "mujer-casa, hombre- 
empresa", o lo que también podría llamarse espacio privado para ellas, espacio público para ellos. Los hombres suelen ser los que encabezan las actividades importantes y prestigiosas, las cuales están fuera de las cuatro paredes del hogar. Es el espacio público, al que pertenecen los varones, el que tiene un valor, mientras que el espacio privado, que carece de valía e importancia ante la mirada social, corresponde a la mujer. Es allí donde le toca a la mujer desarrollar su rol de madre.

Una de las razones con las que se justifica esta separación, es con el discurso referente a que la mujer es inferior al hombre y no tiene las mismas capacidades que este último, por lo que les toca ser cuidadoras del hogar, a pesar de los resultados de rendimiento académico y laboral que demuestran que ambos sexos son igual de capaces (Ramos, Barberá y Sarrió, 2003).

Como menciona Amorós (1994), el espacio público conlleva reconocimiento, por lo tanto, poder. Bajo esta misma lógica, el espacio privado es invisible, lo que implica sumisión. Mientras los hombres están fuera, ejerciendo el poder, las mujeres están dentro, limitadas a procrear y cuidar de sus hijos. Ante esto, Pina refiere que:

Si pensamos en el espacio doméstico y el modo en que es administrado, qué poderes se otorgan, a quiénes se le otorgan, y qué límites se imponen, se hace evidente que el productor de la Ley es el padre y que el poder de juzgar se le ha otorgado a él, o a cualquiera que sea la forma que la voz masculina tome: los vecinos, las costumbres, el juez, el discurso médico, la madre del padre, los discursos especializados sobre maternidad. La mujer es la cabeza del espacio doméstico, pero su comportamiento es legislado, juzgado y castigado desde el exterior; es decir [...] desde el espacio extradoméstico. Ella es la responsable de todo lo que ocurre en el hogar, y debe ser buena en eso, "como una buena madre", pero no puede, bajo ninguna circunstancia, romper los acuerdos sociales; de lo contrario, su poder de representación, el único que posee, corre el riesgo de ser revocado (2006, pp. 130-131).

La dicotomía espacio privado - espacio público es el resultado de una estructura social patriarcal en donde la mujer alcanza su "éxito" desarrollándose en el ámbito doméstico-materno. Cabe resaltar que la maternidad genera beneficios económicos y fomenta que la mujer se quede en casa, permitiendo que el hombre sea el principal ocupante del espacio laboral.

\section{Legitimación de la maternidad desde el conocimiento experto-patriarcal}

La teoría feminista es una teoría de poder, como tal, su objetivo es la explicación del origen de la opresión, supresión y represión de la mujer. El feminismo pretende visibilizar cómo a través de los períodos históricos, distintas ciencias y distintas instituciones legitiman lo que es válido:

Medicina y psicología: Las explicaciones dadas anteriormente, avaladas por las instituciones científicas y por el patriarcado, hacen referencia a que la mujer, con la capacidad natural reproductiva que tiene, está "diseñada” para cumplir con el principal objetivo que 
es ser madre, lo que lleva a la conclusión de que aquellas mujeres que deciden no serlo serán catalogadas como antinaturales. Daniluk describe que la medicina y la psicología, también juegan un rol importante al promover el embarazo y la maternidad como curas a trastornos menstruales, la apoplejía, varias formas de cáncer y enfermedades cardiacas:

[Los médicos] persuaden a las mujeres "infértiles" para que dejen que sus cuerpos sean sometidos durante años a experimentaciones y pruebas médicas, con la esperanza de alcanzar algún día al hijo esperado. [...] de acuerdo con los teóricos del campo de la psicología, las mujeres sin hijos son caracterizadas como egoístas, narcisistas y proclives a sufrir por una "incompletud de su feminización" [...] la ambivalencia materna es considerada patológica, ya que la mujer está negando los "impulsos" naturales y no está cumpliendo con su "verdadero objetivo" de vida (1999, p. 81-82).

Campbell (1999) realizó un estudio con 23 mujeres que no querían tener hijos y que, cansadas de utilizar métodos anticonceptivos o de preocuparse por un embarazo no deseado, decidieron esterilizarse. Uno de los resultados obtenidos en la investigación de Campbell corresponde a la interacción médico-mujer, en la que el profesional de la salud llegaba incluso a negar el procedimiento puesto que, bajo el propio juicio del médico o médica, la mujer podía arrepentirse, retractarse y lamentar su decisión. La medicina utiliza este eufemismo o la falsa premisa de un posible arrepentimiento, cuando en realidad lo que está en juego es el manejo del cuerpo de la mujer a través de controlar su fertilidad.

Dentro del campo de la psicología, Woolet (1991) hace referencia a que algunos psicoterapeutas suelen percibir la vida de las mujeres sin hijos como vacía, carente de satisfacción y consideran que no tienen la habilidad para comprometerse en relaciones cercanas e íntimas. Para concluir con la psicología que refuerza el discurso de la maternidad, Benedek menciona que para algunos psicoterapeutas, "las mujeres tienen mayor oportunidad de lograr su madurez psíquica y emocional cuando aceptan la maternidad que cuando la rechazan" (1978, p. 60).

Cuando la medicina y la psicología no tienen perspectiva de género, pueden llegar a reproducir las prácticas patriarcales en donde se descalifica, con todo el poder que estas ciencias ejercen, el deseo y la opinión de la mujer: "te vas a arrepentir, algún día querrás ser madre", "todas las mujeres quieren ser madres", "estás muy joven para decidir".

Biología: Desde una postura biologicista, Lerner comenta que:

Una explicación corolaria de la asimetría sexual es la que sitúa las causas de la subordinación femenina en factores biológicos que atañen a los hombres. La mayor fuerza física de éstos, su capacidad para correr más rápido y cargar mayor peso, junto con su mayor agresividad, les capacitan para ser cazadores. Por tanto, se convierten en los que suministran los alimentos a la tribu, y se les valora y honra más que a las mujeres. Las habilidades derivadas de las actividades cinegéticas les dotan a su vez para ser guerreros. El hombre cazador, superior en fuerza, con aptitudes, junto con la experiencia nacida del uso de útiles y armas, protege y defiende «naturalmente» a la mujer, más vulnerable y cuya dotación biológica la destina a la maternidad y a la crianza de los hijos (1986, p.24). 
Esta visión ha sido aceptada en su gran mayoría por las bases "científicas" que la sustenta y porque además explica los roles de género y el domino del hombre sobre la mujer de forma tal que exime al hombre de toda responsabilidad, al recaer el peso en cuestiones fisiológicas e innatas, sobre las cuales el ser humano no suele tener control ni un manejo consciente, como lo es la masa muscular, el tipo de cuerpo que permite una mayor agilidad, la producción de testosterona que puede generar altos niveles de agresividad, propiciando, en conjunto, que el hombre sea el que se encargue de cazar y proveer, mientras que la biología femenina propicia el espacio para la creación de un ser humano nuevo, por lo tanto, su cuerpo está preparado para cuidarlo, alimentarlo, protegerlo, etcétera.

Antropología: Ortner (1974) propone una posible explicación a este hecho a partir de la dicotomía cultura-naturaleza. Menciona que los seres humanos se caracterizan y se diferencian de otras especies gracias a la capacidad de crear cultura e ir más allá de la naturaleza. La cultura es algo único de nuestra especie y, por lo tanto, se le atribuye un valor especial. Bajo esta premisa, la naturaleza no es exclusiva del ser humano, así que se le asigna un valor secundario. Debido al rol principalmente materno que se le ha consignado a la mujer, es vista como un ser más cercano a la naturaleza (puesto que no es la única especie que puede procrear) en comparación con el hombre. Así, la mujer tiene un rol secundario e inferior al igual que la naturaleza, mientras que la cultura, que es única del ser humano, se le otorga al hombre y éste se engrandece.

Religión: la religión también tiene su propia explicación del porqué la mujer es inferior al hombre. La mujer está subordinada al hombre porque así lo dispuso Dios. Ante esto, La Biblia ${ }^{2}$ dice que la mujer fue creada a partir de la costilla del hombre, así como también está escrito que la mujer casada está atada por ley a su esposo (Romanos 7:2, 3). Dentro del razonamiento tradicional, Lerner menciona que "puesto que a la mujer se le asignó por designio divino una función biológica diferente a la del hombre, también se le deben adjudicar cometidos sociales distintos" (1986, p. 23).

Por su parte, Hipp realiza un análisis sobre el poder y la influencia que tiene la iglesia y comenta que, de la mano con el Estado, ambas instituciones crean el ideal de la estructura familiar-social y delimitan que "el hombre y la mujer se complementan y se realizan muy especialmente en el plano sexual, siendo el matrimonio el único estado válido para alcanzar la plena realización, pero con un fin procreador" (Hipp, 2006, p. 60)

Estado: Por otro lado, el Estado atraviesa el cuerpo de la mujer con las leyes que prohíben el aborto. En nuestro país, el aborto "libre" (por cualquier motivo) solamente es legal hasta la semana 12 de gestación en dos estados. En el resto del país, existen condicionantes para poder llevarlo a cabo, principalmente si es porque el embarazo fue resultado de una violación. ${ }^{3}$

Cabe resaltar que, en el Código Penal para el Estado de Tamaulipas, se expone que el aborto es causal de hasta cinco años de cárcel, sin embargo, el juez puede sustituir la

\footnotetext{
${ }^{2}$ Ver: Efesios 5:21-27, Génesis 2:23, Génesis 3:16, Génesis 4:1, Génesis 30: 22 Éxodo 23:7, entre otros.

${ }^{3}$ Aunque el embarazo haya sido producto de una violación y la mujer o niña desee realizarse un embarazo, han existido varios casos en donde el estado le prohíbe a la persona el aborto, a pesar de que por ley tenga el derecho a llevarlo a cabo.
} 
sentencia por tratamiento médico integral que implique la reafirmación de los valores humanos por la maternidad y la familia:

Artículo 357: A la mujer que voluntariamente procure su aborto o consienta en que otro la haga abortar, se le impondrá una sanción de uno a cinco años de prisión, quedando facultado el juez para sustituirla por tratamiento médico integral, para lo cual sólo bastará que lo solicite y ratifique la responsable. El tratamiento referido en este precepto será provisto por las Instituciones de Salud del Estado y tendrá como objeto apoyar a las mujeres a superar los efectos causados como consecuencia del aborto provocado, así como reafirmar los valores humanos por la maternidad ayudando al fortalecimiento de la familia. No se concederá el beneficio de sustituir la sanción privativa de libertad por el de tratamiento médico integral, a la mujer que reincida en la comisión del delito de aborto. (Cap. VII, 2016).

Como se puede observar con las leyes, el Estado en relación directa con los valores familiares tradicionales, impuestos principalmente por la religión católica, impide y penaliza a la mujer que toma acción para evitar ser madre una vez que está embarazada.

Las instancias mencionadas en este apartado corresponden al conocimiento experto. Éstas crean e implementan las verdades dominantes que llegan a regir la sociedad (Castillo, Ledo y Pino, 2012). Como menciona Payne (2002), "el poder no se establece por medio de la amenaza o la fuerza, sino por la defensa del conocimiento del especialista que lo instaura en primer lugar y por la oculta persuasión que mueve a la gente a "interiorizar" su inferioridad haciendo de ella una "verdad" (p. 55). En este caso, las "verdades dominantes" se pueden identificar en los principales estereotipos que rodean a la mujer: madre y buena madre.

McIntosh (2013) refiere que, durante los siglos pasados e inclusive en pleno siglo XXI, la maternidad se ha documentado principalmente a partir de los ojos de los hombres. Mientras los hombres tenían oportunidades de estudiar medicina y ser obstetras, podían opinar sobre el cuerpo de las mujeres e implementar la idea de que la maternidad es natural y toda "dama respetable" debe pasar por esta etapa. Explica además que las mujeres que se atrevían a intervenir en el campo de la salud materna, específicamente las parteras, eran consideradas como personas que estaban involucradas en la brujería, magia y superstición. De igual manera se les relacionaba con el aborto y el infanticidio.

Por otro lado, Zemon (1976), expone la importancia de entender los simbolismos, al igual que los significados de los sexos y los géneros a través de la historia, para que de esta manera se pueda comprender el alcance que los roles de género tienen en las distintas sociedades y cómo estos operan de tal forma que mantienen el homeostato social. Por lo tanto, las ciencias e instituciones que refuerzan la práctica materna, lo hacen, además de utilizando el discurso digital, a través de legislaciones, privando a las mujeres de información anticonceptiva, creando tecnologías para la reproducción asistida, enseñando que las relaciones sexuales son solamente para procrear, entre otras.

El psicólogo estadounidense Bronfenbremer (1979) propone la teoría ecológica de los sistemas, la cual consiste en que el individuo se desarrolla cognitiva, emocional, moral y socialmente a través de la influencia de los distintos ambientes o sistemas en los que se desenvuelve: familia, escuela, trabajo, amistades, religión, política, etcétera. Estos sistemas 
rodean al sujeto y están organizados de menor a mayor globalidad, siendo el más directo a la persona el microsistema (familia, vecinos, amistades, compañeros, profesores, etcétera). Después se encuentra el mesosistema, donde se plantean las relaciones entre los distintos elementos que conforman al microsistema (por ejemplo, la relación entre los padres de familia y los profesores de la escuela). Más adelante aparece el exosistema que concierne a las instituciones educativas, de salud, religiosas, políticas, los medios de comunicación y la economía. Para finalizar, está el macrosistema, constituido por la cultura, la moral, las costumbres y las tradiciones. Considerando la teoría de Bronfenbremer, la construcción de la maternidad y la legitimización del discurso materno se crea dentro del exosistema y del macrosistema, ya que el significado social de ésta se gesta a partir de los discursos de los distintos aparatos hegemónicos mencionados en este capítulo, así como con las costumbres y tradiciones de la cultura que se transmiten de generación en generación.

Estas instituciones que crean y respaldan al conocimiento experto, juegan a favor del patriarcado, estableciendo y promoviendo mitos y creencias en torno a ser madre, que son aceptadas por la población debido a que están avaladas científicamente. Foucault (1983) escribe sobre cómo este conocimiento científico controla el cuerpo de la mujer y lo encamina hacia la maternidad. El término que utiliza para explicar la forma en que la ciencia coacciona a la mujer es "biopoder". Fernández lo describe como "un dispositivo disciplinario que se aplica a los cuerpos para vigilarlos, controlarlos, o explotarlos" (2004, p. 191). De igual manera, Fernández (2004) menciona que los cuerpos son controlados, observados e intervenidos, ya que de esta manera se obtienen distintos beneficios para los grupos sociales que se encuentran en ventaja. Desde esta perspectiva, se considera el cuerpo de la mujer como una máquina de producción: produce placer, riquezas, bienes materiales, individuos que más adelante serán partícipes (víctimas, lo más probable) del modelo socioeconómico, fuerza de trabajo barata, así como labores domésticas gratuitas. Foucault expone que el poder "se ejerce sobre la vida cotidiana inmediata que clasifica a los individuos en categorías" (1988, p. 7), por ejemplo "madres" o "no madres". A fin de cuentas, el poder es una forma de dominación y como tal, se domina a la mujer cuando es controlada su reproducción sexual y su planificación familiar.

Los sistemas que legitiman los discursos referentes a la maternidad y que invalidan a las mujeres que deciden no ser madres, son encabezados y manejados principalmente por hombres, quienes reproducen ideologías poderosas que terminan insertándose como normativas sociales.

\section{Las mujeres "No-Mo"4}

Al dejar en claro que la maternidad no es puramente biológica y mucho menos innata, sino que está constituida por factores socio-culturales y psicológicos, es entendible que existan mujeres que están separando sus cuerpos del Estado, de la religión, así como de los

${ }^{4}$ Mujeres No-Mothers. 
discursos médicos y han decidido no procrear. Son mujeres que ya no permiten que los hombres decidan sobre su vida sexual ni que sean ellos quienes controlen la planificación familiar. Están dejando de lado las prácticas masculinas que operan sobre el cuerpo de la mujer y comienzan a decidir por sí mismas si desean o no entrar al ejercicio de la maternidad.

Actualmente, al grupo de mujeres que ha decidido no ser madre, se les conoce como "No-Mo5". Lipovetsky (1999) propone tres categorías para definir a las mujeres: la primera mujer, quien sufre el dominio social del hombre sobre ella; la segunda mujer, que es adorada, engrandecida y sacralizada; y la tercera mujer. Es la tercera categoría la que se asimila con el concepto de mujeres "No-Mo", pues en ésta se encuentran las mujeres que deciden por sí mismas cómo van a experimentar su vida sexual y reproductiva y se enfocan en lo que ellas desean y no en lo que se ha dictado como "normal" a través de la historia.

Hoy en día, hay grupos de mujeres que se han atrevido a transgredir los roles y los estereotipos de género impuestos clásicamente, siendo uno de ellos la maternidad y la familia tradicional. Dever y Saugeres (2004) presentan algunas características sociodemográficas de estas mujeres: pertenecen a una clase social media y media-alta, con niveles altos de educación, que trabajan y tienen una profesión. Welti (en Ávila, 2005) expone, sin mencionar motivos, que en México una de cada diez mujeres decide no ser madre, aventurándose a crear y entrar a distintas estructuras familiares.

Por su parte, Ávila (2005) elabora una lista de varios motivos que han dado las mujeres para decidir no tener hijos: no sienten el apoyo de una pareja o de la familia para cuidar y mantener tanto al hogar como al hijo, no fueron criadas bajo el paradigma de la familia tradicional, perciben el futuro económico y social con incertidumbre y de una manera trágica, la maternidad representa ataduras y el no tener hijos representa libertad para emprender sus sueños y, por último, porque no lo desean y no está en sus planes.

Salirse de la norma puede representar un riesgo y es común que sean discriminadas, etiquetadas, presionadas en los distintos sistemas a los que pertenecen. Ante esto, Lagarde (1990) y Morell (1994), citadas en Ávila (2005), enfatizan cómo las mujeres que no tienen hijos, además de ser coaccionadas y discriminadas en las distintas formas que se han descrito a lo largo de este capítulo, tampoco poseen un espacio propio dentro del mismo lenguaje:

El hecho de carecer de un concepto positivo específico para definir a las mujeres que eligen voluntariamente no ser madres, y tener que describirlas desde el prefijo adjetivado, que enfatiza la falta, la ausencia o la negación, al referirse a las mismas como "mujeres sin hijos", "mujeres no madres", nos habla del nulo lugar que ocupan. Es decir, existe un concepto para definir a las solteras, a las viudas, a las divorciadas, a las lesbianas, pero las mujeres sin hijos no tienen un nombre y un lugar propio, existen desde lo que no son o no tienen, son por tanto algo incompleto, liminal, ambiguo o raro (2005, p. 117).

Con todos estos tipos de discriminación, se busca de manera implícita que la misma mujer se autoculpabilice por no seguir la norma social, sin embargo, aunque representen

\footnotetext{
${ }^{5}$ Se sugiere ver el documental "Las 'No-Mo': las mujeres que no quieren ser mamás". Se encuentra en el siguiente hipervínculo https://www.youtube.com/watch?v=Nd9Jxp2bSpI
} 
un cambio en la estructura familiar tradicional, este grupo de mujeres en vez de enfocarse en tener hijos y cumplir el rol de madre, está buscando oportunidades para autorrealizarse fuera de la maternidad.

\section{Conclusiones}

México, al ser un país tan cercano a la religión católica, aún mantiene los valores conservadores que forjan a la familia. La iglesia, de la mano con el Estado y con algunas instituciones educativas, luchan por la preservación de la estructura familiar tradicional. Estas instituciones desempeñan una función moralizadora al momento de hablar sobre la práctica materna, avalando el estereotipo de la mujer-madre y utilizando la maternidad como un dispositivo de control sobre el cuerpo de la mujer.

A partir de los autores revisados, se concluye que la cultura impone un modelo patriarcal en el cual la mujer tiene la función de reproducción, no obstante, se puede observar que la maternidad no es puramente biológica, sino que es un fenómeno que conlleva aspectos sociales, culturales, contextuales y psicológicos. Al estar en constante interacción con el arquetipo de la "madre", pero, sobre todo, la "buena madre", muchas mujeres no se detienen a reflexionar y analizar si en verdad desean es ser madres, o si, por el contrario, es una expectativa externa que está sumamente arraigada en la construcción de la mujer.

Las mujeres son consideradas como las que tienen el deber de procrear, mientras que no sucede así con los hombres, es por esto que las mujeres que no quieren tener hijos, son más señaladas, juzgadas, cuestionadas, criticadas que los hombres que no lo desean (Hernández, 2019). Durante el proceso de la revisión bibliográfica, la autora del presente artículo en ningún momento encontró teoría que dictara que la paternidad es un asunto biológico, mucho menos que fuera una cuestión con la cual el hombre consigue el éxito y se auto realiza, premisas sobre la mujer que sí existían en una gran cantidad de artículos revisados.

\section{Bibliografía}

AMORÓS, CELIA (1994). Espacio público, espacio privado y definiciones ideológicas de 'o masculino' y 'lo femenino'. México: UNAM.

ÁVILA, YANINA (2005). "Mujeres frente a los espejos de la maternidad: las que eligen no ser madres". México: Desacatos, núm. 17, pp. 107-126.

BENEDEK, THERESE (1978). Psychobiological aspects of motherning in psychoanalytic investigation. United States of America: Quadrangle.

BERGER, PETER, LUCKMANN, THOMAS (1968). La construcción social de la realidad. Argentina: Amorrortu.

BRONFENBREMER, URIE (1979). The ecology of human development. United States of America: Harvard University Press. 
CAMPBELL, ANNILY (1999). Childfree and sterilized. Women's decisions and medical responses. United States of America: Cassell.

CASTILLO, IVÁN, LEDO, HILDA, PINO, YASIEL (2012). “Técnicas narrativas: un enfoque psicoterapéutico”. España: Norte de salud mental, núm. 42, pp. 59-66.

DANILUK, JUDITH (1999). "When biology isn't destiny: implications for the sexuality of women without children". Canada: Canadian Journal of counselling, num. 33, pp. 79-93.

DEVER, MARYANNE, SAUGERES, LISE (2004). "I forgot to have children! Untangling links between feminism, careers and voluntary childlessness". Journal of the motherhood initiative for research and community involvement, num. 2, pp. 116-126.

FERNÁNDEZ, INÉS (2004). "Medicina y poder sobre los cuerpos”. España: Themata, revista de filosofía, núm. 33, pp. 191-198.

FOUCAULT, M. (1983). En Dreyfus, H. y Rabinow, P. Más allá del estructuralismo y la hermenéutica. Estados Unidos de América: Chicago University Press.

FOUCAULT, MICHEL (1988). "El sujeto y el poder". Revista mexicana de sociología, núm. 3, pp. 3-20. Recuperado de http://terceridad.net/wordpress/wp-content/ uploads/2011/10/Foucault-M.-El-sujeto-y-el-poder.pdf

HARDING, SANDRA (1983). Why has the Sex/Gender system become visible only now? United States of America: D. Reidel Publishing Coop.

HERNÁNDEZ, JÉSSICA (2019). Parejas que no quieren tener hijos: narrativas y discursos dominantes sobre la maternidad y la familia tradicional en México (tesis de maestría). Instituto Bateson de Psicoterapia Sistémica. México.

HIPP, ROSWITHA (2006). Orígenes del matrimonio y de la familia modernos. España: Universidad Autónoma de Madrid.

LAGARDE, MARCELA (1990). Los cautiverios de las mujeres: madresposas, monjas, putas, presas y locas. México: Universidad Nacional Autónoma de México.

LAMAS, MARTA (1986). "La antropología feminista y la categoría de género". Revista Nueva Antropología, núm. 8, pp. 173-198.

LERNER, GERDA (1986). The Creation of Patriarchy. United States of America: Oxford University Press.

LIPOVETSKY, GILLES (1999). La tercera mujer. España: Anagrama.

MCINTOSH, TANIA (2013). A social history of maternity and childbirth: key themes in maternity care. United States of America: Routledge.

MORELL, CAROLYN (1994). Unwomanly conduct: the challenges of intentional childlessness. United States of America: Routledge.

ORTNER, SHERRY (1974). Is female to male as nature is to culture? United States of America: Standford University Press.

PALOMAR, CRISTINA (2005). “Maternidad: historia y cultura”. México: Revista de estudios de género La Ventana, núm. 22, pp. 35-67.

PAYNE, MARTIN (2002). Terapia narrativa: una introducción para profesionales. España: Paidós. 
PINA, GUADALUPE (2006). "La literatura como espacio de resistencia. Mujer y maternidad: la falacia del espacio privado”. Perú: Revista de crítica literaria latinoamericana, núm. 62, pp. 125-142.

RAMOS, AMPARO, BARBERÁ, ESTER, SARRIÓ, MAITE (2003). “Mujeres directivas, espacio de poder y relaciones de género". España: Anuario de psicología, núm. 34, pp. 267-278.

SALETTI, LORENA (2008). "Propuestas teóricas feministas en relación al concepto de maternidad”. Revista Clepsydra, núm. 7, pp. 169-183.

WOOLLET, ANNE (1991). Motherhood: Meanings and Practices. England: Sage.

ZEMON, NATALIE (1976). "Women's history in transition: the European case”. United States of America: Feminist studies, num. 3, pp. 83-103. 